\title{
Estimating Grid-Induced Errors in CFD by Discrete-Error-Transport Equations
}

\author{
Y. Qin, ${ }^{1}$ P.S. Keller, ${ }^{2}$ R.L. Sun, ${ }^{2}$ E.C. Hernandez, ${ }^{3}$ C.-Y. Perng, ${ }^{3}$ \\ N. Trigui, ${ }^{3}$ Z. Han, ${ }^{3}$ F.Z. Shen, ${ }^{4}$ T. Shieh, ${ }^{5}$ T.I-P. Shih ${ }^{6}$ \\ ${ }^{1}$ Department of Mechanical Engineering, Michigan State University, East Lansing, Michigan 48824 \\ ${ }^{2}$ DaimlerChrysler, Auburn Hills, Michigan 48326 \\ ${ }^{3}$ Ford Motor Company, Dearborn, Michigan 48121 \\ ${ }^{4}$ General Motors Corporations, Flint, Michigan 48557 \\ ${ }^{5}$ Toyota Technical Center, Ann Arbor, Michigan 48103 \\ ${ }^{6}$ Department of Aerospace Engineering, Iowa State University, Ames, Iowa 50011
}

\begin{abstract}
This paper is dedicated to the memory of Derlon Chu (Nov. 20, 1959 - June 19, 2003) - a colleague and a friend who has contributed much to make CFD impact the design of automotive components. Derlon Chu spearheaded the formation of the "Partnership on CFD Codes and Models for the Automotive Industry" to address error issues in CFD.
\end{abstract}

\begin{abstract}
This paper presents and evaluates a method for estimating grid-induced errors in CFD solutions that recognizes error at one location in the flow domain may not be generated there, but rather generated elsewhere and then transported there. This paper derives a system of discrete error-transport equations (DETEs) to compute the evolution of grid-induced errors in finite-volume solutions of the Euler equations for compressible flows in two dimensions. The finite-volume method to which the DETEs were derived is one which can be applied to structured or unstructured meshes with cells that can be triangular, rectangular, or other polygons. Results for a test problem involving an oblique shock wave show that if the residuals in the DETEs are modeled accurately, then the DETEs can predict grid-induced errors accurately.
\end{abstract}

\section{INTRODUCTION}

With computational fluid dynamics (CFD) becoming more accepted and more widely used in industry for design and analysis, there is increasing demand for not just more accurate solutions, but also error bounds on the solutions. The error in each CFD solution can be attributed to three main sources (see, e.g., Roache ${ }^{1}$, Oberkampf, et al. ${ }^{2}$, and Cosner, et $\mathrm{al}^{3}{ }^{3}$ ). The first is inadequate modeling of physics that are not resolved by first principles such as turbulence inside the flow domain and inflow boundary conditions at the domain boundary. The second is nonphysical effects such as numerical diffusion and dispersion that result when the governing partial differential equations (PDEs) are discretized into algebraic equations on a discrete domain by finite-difference, finite-volume, or finite-element methods. The third source of error is from a poor quality or an insufficiently fine grid or mesh.

Copyright (C) 2004 by T. I-P. Shih. Published by the American Institute of Aeronautics and Astronautics, Inc. with permission.
For most users of CFD, especially those who do not have access to the source code, the mesh and the time-step size are the only parts of the solution procedure in which the user has full control. The importance of the mesh cannot be over emphasized. The mesh must represent the geometry with sufficient detail and enable the algebraic analog of the governing PDEs to resolve the relevant flow physics. For complicated steady and unsteady, threedimensional problems, the number of grid points or cells that can be used in a mesh is restricted by either the available computer resource or a need to have a practical turn-around time in computing a solution. With a constraint on the number of grid points or cells, accuracy demands grid points to be placed in regions where they are most needed to resolve the geometry and flow physics (e.g., by $\mathrm{r}$ - or h-refinement). Unfortunately, this nonuniform distribution can create what are referred to as poor-quality cells, which can induce considerable errors in the computed solutions ${ }^{4}$. Also, even when using the solution to adapt the grid as optimally as possible, the solution generated is often far from being grid independent because of resource constraints. 
Thus, the questions are (1) what can one believe in the computed solutions? (2) What is the error bound on the solutions from a poor-quality or an insufficiently fine grid? How can the grid or mesh be improved to increase accuracy?

\section{PREVIOUS WORK}

A number of investigators have developed and evaluated ways to quantify errors in solutions of PDEs that arise from poor-quality or insufficiently fine grids/meshes. Roache $^{1}$ reviewed and classified all of these methods into two categories: methods based on multiple grids and methods based on a single grid. Methods based on multiple grids ${ }^{5-7}$ require solutions to be generated on a series of increasingly finer grids (at least 3). These methods can give definitive statements on errors, but is more expensive in requiring solutions at finer meshes, which can be prohibitive for realistic engineering problems. If Richardson's extrapolation is used, then the meshes must be sufficiently fine for the truncated Taylor series to be bounded before this method can yield meaningful results.

Single-grid error-estimation methods can be classified as algebraic or PDEs. Algebraic methods assume that the error at a grid point or cell is a function of the grid in question and the solution there. A lot of work has been done on algebraic methods, mostly in relation to solutionadaptive mesh refinement (see, e.g., Carey ${ }^{4}$, and Refs. 8 to 14). These algebraic error estimators, also referred to as grid-quality measures, typically only consider gradients of the scalar fields. Shih, et al. ${ }^{15}$ and $\mathrm{Gu}$, et al. ${ }^{16,17}$ proposed grid-quality measures that also account for the vector and tensor nature of the flow field and link the solution to the geometry and size of each cell in a grid.

Single-grid PDE error estimators, first proposed by Babuska, et al. ${ }^{18,19}$, recognize that errors once generated can be transported by advection and diffusion to other parts of the flow field. Thus, a transport equation is needed to understand the generation and evolution of errors.

A method for deriving transport equations for error was presented by Babuska, et al. ${ }^{18,19}$ for finite-element methods. Ferziger ${ }^{20}$, Van Straalen, et al. ${ }^{21}$, and Zhang, et al. $^{22,23}$ applied it to finite-difference and finite-volume methods. Their method proceeds as follows. Consider a differential operator L operating on dependent variable U:

$$
\mathrm{L}(\mathrm{U})=\mathrm{f}
$$

where $f$ contains the non-homogeneous terms. If $\mathrm{U}_{\mathrm{a}}$ is an approximate solution, then its substitution into Eq. (1) will produce a residual $\mathrm{R}$ since it will not satisfy Eq. (1); i.e.,

$$
L\left(U_{a}\right)-f=R
$$

If Eqs. (1) and (2) are linear or linearized, then subtracting Eq. (2) from Eq. (1) yields the following transport equation for error:

$$
\mathrm{L}(\mathrm{e})=-\mathrm{R} \text { or } \mathrm{L} \mathrm{e}=-\mathrm{R}
$$

where

$$
\mathrm{e}=\mathrm{U}-\mathrm{U}_{\mathrm{a}}
$$

is the solution error. Since the error in the solution is defined with respect to the exact solution of the PDE, the error-transport equation given by Eqs. (3) and (4) can account for both errors from the grid and errors from the numerical scheme.

Qin and Shih ${ }^{24}$ noted that Eqs. (3) and (4) is only valid for finite-expansion methods such as finite element and spectral, but not for collocation-type methods such as finite-difference (FD) and finite-volume (FV). This is because for FD and FV methods, the differential operator $\mathrm{L}$ in Eq. (2) is replaced by a discrete operator so that subtracting Eq. (2) from Eq. (1) will not yield Eqs. (3) and (4). This inconsistency was also noted by Roache ${ }^{1}$. To rectify this inconsistency, Roache ${ }^{1}$ suggested all FD and FV solutions at grid points or cells to be made into continuous functions. However, Roache ${ }^{1}$ recognizes that the resulting continuous function is non-unique and will depend on the interpolants used.

Qin and Shih $^{24}$ proposed to overcome the inconsistency by defining a discrete-error-transport equation that is based on the FD/FV method without regard for the original PDE. Their method proceeds as follows. Suppose for a FD/FV method, the differential operator L in Eq. (1) is replaced by the discrete operator $\mathrm{L}_{\mathrm{D}}$ so that the algebraic analog of Eq. (1) is

$$
\mathrm{L}_{\mathrm{D}}\left(\mathrm{U}_{\mathrm{i}}, \mathrm{h} . \mathrm{k}\right)=0
$$

where $U_{i}$ is the solution at grid point or cell $i$; h denotes the grid spacing or cell size; and $\mathrm{k}$ denotes the time-step size. As $h$ and $k$ are refined for an unconditionally consistent FD/FV equation, the solution $U_{i}$ approaches the gridindependent solution $\mathrm{U}_{\mathrm{g}, \mathrm{i}}$. With Eq. (5), the gridindependent solution is given by

$$
\mathrm{L}_{\mathrm{D}}\left(\mathrm{U}_{\mathrm{g}, \mathrm{i}}, \mathrm{h}_{\mathrm{g}}, \mathrm{k}_{\mathrm{g}}\right)=0
$$

where $h_{g}$ is the grid spacing or cell size and $k_{g}$ is the timestep size needed to obtain the grid-independent solution. Qin and $\mathrm{Shih}^{24}$ derived two different discrete-errortransport equations from Eqs. (5) and (6). One is based on the coarse grid spacing $h$, and the other is based on the grid-independent grid spacing $h_{g}$. They showed that only the one based on the coarse grid spacing has meaning because one can accurately interpolate $U_{g, i}$ onto $h$ but not $\mathrm{U}$ onto $\mathrm{h}_{\mathrm{g}}$. Thus, following Qin \& $\mathrm{Shih}^{24}$, the grid- 
independent solution is inserted into Eq. (5) with the coarse mesh $(\mathrm{h}, \mathrm{k})$. This produces a residual; i.e.,

$$
\mathrm{L}_{\mathrm{D}}\left(\mathrm{U}_{\mathrm{g}, \mathrm{i}}, \mathrm{h}, \mathrm{k}\right)=\mathrm{R}_{\mathrm{i}}
$$

If $\mathrm{L}_{\mathrm{D}}$ is linear or linearized, then substracting Eq. (5) from Eq. (7) gives the discrete-error-transport equation, which is

$$
\begin{aligned}
& L_{D}\left(e_{i}, h, k\right)=R_{i} \text { or } L_{D}(h, k) e_{i}=R_{i} \\
& e_{i}=U_{g, i}-U_{i}
\end{aligned}
$$

Since $U_{g, i}$ is not unique in the strict sense and may differ from the exact solution because of spurious modes permitted by the FD/FV method, Eqs. (8) and (9) can only account for grid-induced errors, but not for errors from the FD/FV discretization. This is a deficiency of the discreteerror-transport equation, henceforth referred to as DETE.

Qin \& $\operatorname{Shih}^{24,25}$ showed that if the "actual" residual defined by Eq. (7); i.e.,

$$
\mathrm{R}_{\mathrm{i}}=\mathrm{L}_{\mathrm{D}}\left(\mathrm{U}_{\mathrm{g}, \mathrm{i}}, \mathrm{h}, \mathrm{k}\right) \text { or } \mathrm{R}_{\mathrm{i}}=\mathrm{L}_{\mathrm{D}}(\mathrm{h}, \mathrm{k}) \mathrm{U}_{\mathrm{g}, \mathrm{i}}
$$

is used (which requires the grid-independent solution to be computed), then the DETE given by Eq. (8) can predict grid-induced errors perfectly for FD/FV equations that are linear, nonlinear, steady, or unsteady. They also found that the linearization procedure used to derive DETE for nonlinear FD/FV equations need not be conservative when computing weak solutions because the error-propagation speed is captured by the FD/FV equations (which are in conservative form), and not by the DETE.

In practice, the grid-independent solution is not computed and so the residual in Eq. (8) must be modeled. This is the main challenge when implementing both the continuous and the discrete error-transport equation. The modified equation of Warming \& $\mathrm{Hyett}^{26}$ has been used to guide the modeling of the residual ${ }^{22-25}$. Zhang, et al. ${ }^{2,23}$ and Qin \& Shih ${ }^{24,25}$ showed that modeling the residual by using the leading terms of the truncation error in the modified equation can give excellent results if the grid spacing or cell sizes are sufficiently small. However, when the grid spacing or cell size is too large, then the error predictions can be quite poor. Recently, Celik, et al. ${ }^{27}$ modeled the residual in Eq. (8) by expanding terms in the FD/FV equations about the cell center and keeping the leading terms. This modeling approach is essentially the same as using the modified-equation except timederivatives are not replaced by spatial derivatives.

Since the modified equation and Taylor-series expansions are not useful in modeling the residual when the grid is coarse (the typical situation), Qin \& Shih $^{28}$ proposed an alternative approach based on data mining. This approach involves two steps. The first is to study the behavior of the residual by evaluating the "actual" residual created by a variety of poor quality meshes in a systematic way. The second step is to model the residual based on the understanding gained on the behavior of the residual through statistical analysis and curve fitting.

\section{OBJECTIVE}

So far, the discrete-error transport equation (DETE) has only been developed and demonstrated for simple model equations such as the advection diffusion equations and the inviscid Burger equation. Also, it has only been applied on structured grids, where finite-difference and finite-volume methods can lead to identical algebraic analogs of PDEs. Thus, the objective of this study is to develop a DETE for a more complicated set of equations in the framework of arbitrary shaped elements (e.g., triangles, rectangles, and other polygons).

The remainder of this paper is organized as follows. First, the test problem selected is summarized. Next, the finite-volume method (FVM) enabling solution on arbitrary elements is derived. Then, the DETE corresponding to this FVM is derived. This is followed by some preliminary results.

\section{DESCRIPTION of TEST PROBLEM}

The test problem involves two parts, the set of PDEs and the application problem. The set of PDEs selected is the continuity, $\mathrm{x}^{-}$and $\mathrm{y}$-momentum, and total-energy equations valid for two-dimensional compressible, inviscid flow of a colorically and thermally perfect gas. These equations can be written as

$$
\begin{aligned}
& \frac{\partial Q}{\partial t}+\frac{\partial F}{\partial x}+\frac{\partial G}{\partial y}=0 \\
& Q=\left\{\begin{array}{l}
\rho \\
\rho u \\
\rho v \\
E
\end{array}\right\}, F=\left\{\begin{array}{l}
\rho u^{2}+p \\
\rho u v \\
u(E+p)
\end{array}\right\}, G=\left\{\begin{array}{l}
\rho u v \\
\rho v^{2}+p \\
v(E+p)
\end{array}\right\} \\
& E=\frac{p}{\gamma-1}+\frac{1}{2} \rho\left(u^{2}+v^{2}\right)
\end{aligned}
$$

where $\rho$ is density; $u$ and $v$ are the $x$ - and $y$-components of the velocity, respectively; $\mathrm{E}$ is mechanical and thermal energy; $\mathrm{P}$ is pressure; $\mathrm{T}$ is temperature; and $\gamma$ is the ratio of specific heats.

The application problem selected is uniform supersonic flow with freestream Mach number $M_{1}$ and pressure $\mathrm{P}_{1}$ over a sharp edge wedge with half angle $\theta$. A schematic diagram is shown in Fig. 1. As can be seen in the figure, only half of the wedge is simulated because of 
symmetry. For this problem, there is an exact solution given by

$$
\begin{aligned}
& \frac{1}{\tan \alpha}=\left[\left(\frac{\gamma+1}{2}\right)\left(\frac{M_{1}^{2}}{M_{1}^{2} \sin ^{2} \theta}\right)-1\right] \tan \theta \\
& \frac{P_{2}}{P_{1}}=\left(\frac{2 \gamma}{\gamma+1}\right) M_{1}^{2} \sin ^{2} \theta-\left(\frac{\gamma-1}{\gamma+1}\right) \\
& \frac{\rho_{2}}{\rho_{1}}=\frac{\tan \theta}{\tan \alpha}=\frac{(\gamma+1) M_{1}^{2} \sin ^{2} \theta}{2+(\gamma-1) M_{1}^{2} \sin ^{2} \theta} \\
& \frac{\left|V_{2}\right|}{\left|V_{1}\right|}=\frac{\sin \theta}{\sin (\theta-\alpha)}\left[\frac{2}{(\gamma+1) M_{1}^{2} \sin ^{2} \theta}+\left(\frac{\gamma-1}{\gamma+1}\right)\right]
\end{aligned}
$$

where $\alpha$ is the angle of the oblique shock with respect to upstream flow direction; 1 denotes the condition upstream of the oblique shock; 2 denotes the condition down stream of the oblique shock; $\mathrm{V}_{1}=\mathrm{M}_{1} \sqrt{\gamma \mathrm{RT}_{1}}(\mathrm{R}$ is the gas constant); $\mathrm{V}_{2}$ is the magnitude of the velocity downstream of the oblique shock. This solution is valid as long as the angle of the wedge $\theta$ is sufficiently small so that a bow shock does not form upstream of the wedge.

The boundary conditions used are specified freestream conditions at the inflow boundary, extrapolated boundary condition at the outflow boundary, zero flux/flow boundary condition at the symmetry plane and the wedge surface.

\section{FINITE-VOLUME METHOD OF SOLUTION}

In this study, a cell-centered, finite-volume method is used to generate solutions to Eq. (11) in which the mesh can be structured or unstructured and the cells can have arbitrary shapes such as triangles, rectangles, or other polygons. The method proceeds as follows. ${ }^{30}$

Integrating Eq. (11a) over an arbitrary i'th cell with volume $\mathrm{V}_{\mathrm{i}}$ in a flow domain replaced by a structured or unstructured mesh gives

$$
\int_{V_{i}}\left(\frac{\partial Q}{\partial t}+\frac{\partial F}{\partial x}+\frac{\partial G}{\partial y}\right) d V=0
$$

or

$$
\begin{aligned}
& \int_{V_{i}}\left(\frac{\partial Q}{\partial t}+\nabla \cdot \vec{f}\right) d V=0 \\
& \vec{f}=F \vec{i}+G \vec{j}
\end{aligned}
$$

where $\vec{i}$ and $\vec{j}$ are unit vectors in the $x$ - and y-directions. By invoking Gauss' theorem,

$$
\int_{V_{i}} \nabla \bullet \vec{f} d V=\int_{\partial V} \vec{f} \bullet \vec{n} d S, \vec{n}=n_{x} \vec{i}+n_{y} \vec{j}
$$

Eq. (13) becomes (assuming cell does not change in time)

$$
\begin{aligned}
& \frac{\partial \overline{\mathrm{Q}}_{\mathrm{i}}}{\partial \mathrm{t}} \mathrm{V}_{\mathrm{i}}+\int_{\partial \mathrm{V}_{\mathrm{i}}} \overrightarrow{\mathrm{f}} \bullet \overrightarrow{\mathrm{n}} \mathrm{dS}=0 \\
& \overline{\mathrm{Q}}_{\mathrm{i}}=\frac{1}{\mathrm{~V}_{\mathrm{i}}} \int_{\mathrm{V}_{\mathrm{i}}} \mathrm{Q} \mathrm{dV}
\end{aligned}
$$

If the boundary $\partial \mathrm{V}_{\mathrm{i}}$ that surrounds cell volume $\mathrm{V}_{\mathrm{i}}$ involves $\mathrm{J}_{\mathrm{i}}$ faces (with the faces being lines in 2-D and planes in 3-D), then Eq. (15) becomes

$$
\begin{aligned}
& \frac{\partial \bar{Q}_{i}}{\partial t}=\Psi_{i} \\
& \Psi_{i}=-\frac{1}{V_{i}} \sum_{j=1}^{J_{i}} \int_{S_{i, j}} \vec{f} \bullet \vec{n} d S=-\frac{1}{V_{i}} \sum_{j=1}^{J_{i}} \bar{f}_{i, j} S_{i, j} \\
& \bar{f}_{i, j}=\frac{1}{S_{i, j}} \int_{S_{i, j}} \vec{f} \bullet \vec{n} d S
\end{aligned}
$$

where $S_{i, j}$ denotes the length of the $j^{\text {th }}$ face.

So far, no approximations has been made. In this study, the mean fluxes on the faces given by Eq. (16c) is approximated by the following first-order Lax-Friedrichs formula, which is TVD preserving,

$$
\begin{gathered}
\overline{\mathrm{f}}_{\mathrm{i}, \mathrm{j}}=\hat{\mathrm{f}}\left(\overline{\mathrm{Q}}_{\mathrm{L}}, \overline{\mathrm{Q}}_{\mathrm{R}}, \overrightarrow{\mathrm{n}}\right)=\left[\mathrm{f}\left(\overline{\mathrm{Q}}_{\mathrm{L}}\right)+\mathrm{f}\left(\overline{\mathrm{Q}}_{\mathrm{R}}\right)\right] \\
\left.-\left(\left|\mathrm{v}_{\mathrm{n}, \mathrm{av}}\right|+\mathrm{c}_{\mathrm{av}}\right)\left(\overline{\mathrm{Q}}_{\mathrm{R}}-\overline{\mathrm{Q}}_{\mathrm{L}}\right)\right] / 2
\end{gathered}
$$

In the above equation, the subscripts $\mathrm{L}$ and $\mathrm{R}$ refer to the left and right cells about face $\mathrm{j} ; \mathrm{v}_{\mathrm{n}, \mathrm{av}}=\left(\mathrm{v}_{\mathrm{n}, \mathrm{L}}+\mathrm{v}_{\mathrm{n}, \mathrm{R}}\right) / 2$ is the average face normal velocity; and $c_{a v}=\left(c_{L}+c_{R}\right) / 2$ is the average speed of sound.

The time derivative in Eq. (16a) is approximated by the following two-stage Runge-Kutta scheme:

$$
\begin{aligned}
& \overline{\mathrm{Q}}_{\mathrm{i}}^{(1)}=\overline{\mathrm{Q}}_{\mathrm{i}}^{\mathrm{n}}+\Delta \mathrm{t} \Psi\left(\overline{\mathrm{Q}}_{\mathrm{i}}^{\mathrm{n}}\right) \\
& \overline{\mathrm{Q}}_{\mathrm{i}}^{\mathrm{n}+1}=\frac{1}{2} \overline{\mathrm{Q}}_{\mathrm{i}}^{\mathrm{n}}+\frac{1}{2} \overline{\mathrm{Q}}_{\mathrm{i}}^{(1)}+\frac{1}{2} \Delta \mathrm{t}_{\mathrm{i}} \Psi\left(\overline{\mathrm{Q}}_{\mathrm{i}}^{(1)}\right)
\end{aligned}
$$

where the solution $\left(\overline{\mathrm{Q}}^{\mathrm{n}}\right)$ at the $\mathrm{n}^{\text {th }}$ time level $\left(\mathrm{t}^{\mathrm{n}}\right)$ is assumed to be known, and the solution $\left(\overline{\mathrm{Q}}^{\mathrm{n}+1}\right)$ at the $(\mathrm{n}+1)^{\text {th }}$ time level $\left(\mathrm{t}^{\mathrm{n}+1}\right)$ is sought.

Since only steady-state solutions are of interest in this study, local time stepping is implemented to accelerate 
convergence rate. The time step size at each cell $\left(\Delta \mathrm{t}_{\mathrm{i}}\right)$ is limited by CFL condition given by

$$
\Delta \mathrm{t}_{\mathrm{i}}=\mathrm{CFL} \mathrm{V}_{\mathrm{i}} / \sum_{\mathrm{j}=1}^{\mathrm{J}_{\mathrm{i}}}\left(\left|\mathrm{v}_{\mathrm{n}}\right|+\mathrm{c}\right)_{\mathrm{j}} \mathrm{S}_{\mathrm{i}, \mathrm{j}}
$$

This completes the finite-volume method employed if only the solution is sought. However, if we also seek to estimate grid-induced errors, then it is important to note that $F$ and $G$ in Eq. (13c) can also be written as

$$
\mathrm{F}=\mathbf{A Q}, \mathrm{G}=\mathbf{B} \mathbf{Q}
$$

where

$$
\begin{aligned}
& A=\frac{\partial F}{\partial Q}= \\
& \left(\begin{array}{cccc}
\frac{\gamma-3}{2} \mathrm{u}^{2}+\frac{\gamma-1}{2} \mathrm{v}^{2} & (3-\gamma) \mathrm{u} & (1-\gamma) \mathrm{v} & \gamma-1 \\
-\mathrm{uv} & \mathrm{v} & \mathrm{u} & 0 \\
(\gamma-1)\left(\mathrm{u}^{2}+\mathrm{v}^{2}\right) \mathrm{u}-\frac{\gamma \mathrm{uE}}{\rho} & \frac{1-\gamma}{2}\left(3 \mathrm{u}^{2}+\mathrm{v}^{2}\right)+\frac{\gamma \mathrm{E}}{\rho} & (1-\gamma) \mathrm{uv} & \gamma \mathrm{u}
\end{array}\right) \\
& B=\frac{\partial G}{\partial Q}= \\
& \left(\begin{array}{cccc}
0 & 0 & 1 & 0 \\
-\mathrm{uv} & \mathrm{v} & \mathrm{u} & 0 \\
\frac{\gamma-3}{2} \mathrm{v}^{2}+\frac{\gamma-1}{2} \mathrm{u}^{2} & (1-\gamma) \mathrm{u} & (3-\gamma) \mathrm{v} & \gamma-1 \\
(\gamma-1)\left(\mathrm{u}^{2}+\mathrm{v}^{2}\right) \mathrm{v}-\frac{\gamma \mathrm{uE}}{\rho} & (1-\gamma) \mathrm{uv} & \frac{1-\gamma}{2}\left(3 \mathrm{v}^{2}+\mathrm{u}^{2}\right)+\frac{\gamma \mathrm{E}}{\rho} & \gamma \mathrm{v}
\end{array}\right) \\
& \frac{\gamma \mathrm{E}}{\rho}=\frac{\mathrm{c}^{2}}{\gamma-1}+\frac{\gamma}{2}\left(\mathrm{u}^{2}+\mathrm{v}^{2}\right)
\end{aligned}
$$

This is because $\mathrm{F}$ and $\mathrm{G}$ are homogeneous functions of $\mathrm{Q}$ to degree one. Thus, there are two ways to evaluate $\mathrm{F}$ and G, via Eq. (11b) or via Eq. (20). Both give identical results. With Eq. (20), f(Q) in Eq. (17) becomes

$$
\mathrm{f}(\mathrm{Q})=\mathrm{AQ} \mathrm{n}_{\mathrm{x}}+\mathrm{BQ} \mathrm{n}_{\mathrm{y}}
$$

Equations (20) and (21) are needed because the DETEs require linearization. Incidentally, these equations are also needed if an implicit method was used to approximate the time derivatives.

\section{DISCRETE ERROR TRANSPORT EQUATIONS}

The derivation of the discrete-error-transport equations (DETEs) for the finite-volume equations (FVEs) given by Eqs. (16) to (21) proceeds as follows. The first step is to linearize the FVEs. As noted by Qin \& Shih, ${ }^{24}$ it is important to linearize by using values of variables computed on the "coarse" mesh for which error estimation is sought. Thus, the linearized FVEs have the following form:

$$
\begin{aligned}
\frac{d \bar{Q}_{i}}{d t}+ & \frac{1}{\left(V_{i}\right)_{c}} \sum_{j=1}^{J_{i}} \frac{1}{2}\left[f_{L}+f_{R}-\left(\left|v_{n, a v}\right|+c_{a v}\right)_{c}\left(\bar{Q}_{R}-\bar{Q}_{L}\right)\right]\left(S_{i, j}\right)_{c}=0 \\
f_{L} & =\left(\left(A_{c} \cdot \bar{Q}\right)_{L} \cdot n_{x}+\left(B_{c} \cdot \bar{Q}\right)_{L} \cdot n_{y}\right) \\
f_{R} & =\left(\left(A_{c} \cdot \bar{Q}\right)_{R} \cdot n_{x}+\left(B_{c} \cdot \bar{Q}\right)_{R} \cdot n_{y}\right)
\end{aligned}
$$

where the time derivative is approximated by Eq. (18). In Eq. (22), all variables with subscript $\mathrm{c}$ are evaluated by using the information on the coarse mesh.

It is important to note that though Eq. (22) is said to have been linearized, it is identical to the non-linearized FVEs because of the identities given by Eq. (20). Thus, when the solution $\mathrm{Q}_{\mathrm{c}}$ obtained on the coarse mesh is inserted into Eq. (22), they will satisfy Eq. (22) perfectly with no residuals. Equation (22) is considered linearized only when the grid-independent solution is inserted into it because in that case the coefficients $\mathrm{A}, \mathrm{B}$, and $\mathrm{V}$ are not evaluated by using the information on the mesh used to generate the grid-independent solution. Thus, when the grid independent solution $\mathrm{Q}_{\mathrm{g}}$ is inserted into Eq. (22), a residual $R_{g}$ will be produced.

Thus, second step is to subtract the Eq. (22) with $Q_{c}$ inserted from the Eq. (22) with the $\mathrm{Q}_{\mathrm{g}}$ inserted. The resulting equation constitutes a system of DETEs for the FVEs given by Eqs. (16) to (21), which is

$$
\begin{gathered}
\frac{\mathrm{de}_{\mathrm{i}}}{\mathrm{dt}}+\frac{1}{\left(\mathrm{~V}_{\mathrm{i}}\right)_{\mathrm{c}}} \sum_{\mathrm{j}=1}^{\mathrm{J}_{\mathrm{i}}} \frac{1}{2}\left[\mathrm{f}_{\mathrm{L}}^{\mathrm{e}}+\mathrm{f}_{\mathrm{R}}^{\mathrm{e}}-\left(\left|\mathrm{v}_{\mathrm{n}, \mathrm{av}}\right|+\mathrm{c}_{\mathrm{av}}\right)_{\mathrm{c}}\left(\mathrm{e}_{\mathrm{R}}-\mathrm{e}_{\mathrm{L}}\right)\right]\left(\mathrm{S}_{\mathrm{i}, \mathrm{j}}\right)_{\mathrm{c}}=\mathrm{R}_{\mathrm{g}} \\
\mathrm{f}_{\mathrm{L}}^{\mathrm{e}}=\left(\left(\mathrm{A}_{\mathrm{c}} \mathrm{e}\right)_{\mathrm{L}} \cdot \mathrm{n}_{\mathrm{x}}+\left(\mathrm{B}_{\mathrm{c}} \mathrm{e}\right)_{\mathrm{L}} \cdot \mathrm{n}_{\mathrm{y}}\right) \\
\mathrm{f}_{\mathrm{R}}^{\mathrm{e}}=\left(\left(\mathrm{A}_{\mathrm{c}} \mathrm{e}\right)_{\mathrm{R}} \cdot \mathrm{n}_{\mathrm{x}}+\left(\mathrm{B}_{\mathrm{c}} \mathrm{e}\right)_{\mathrm{R}} \cdot \mathrm{n}_{\mathrm{y}}\right)
\end{gathered}
$$

where e is the grid-induced error and is given by

$$
\mathrm{e}=\overline{\mathrm{Q}}_{\mathrm{g}}-\overline{\mathrm{Q}}_{\mathrm{c}}=\left\{\begin{array}{l}
\bar{\rho}_{\mathrm{g}}-\bar{\rho}_{\mathrm{c}} \\
\overline{(\overline{\rho u})_{\mathrm{g}}-(\overline{\rho \mathrm{u}})_{\mathrm{c}}} \\
(\overline{\rho v})_{\mathrm{g}}-(\overline{\rho v})_{\mathrm{c}} \\
(\overline{\mathrm{E}})_{\mathrm{g}}-(\overline{\mathrm{E}})_{\mathrm{c}}
\end{array}\right\}
$$

\section{RESULTS}

With the Euler equations summarized, the finitevolume method of solution described, and the discreteerror-transport equations (DETEs) derived, we now 
examine the usefulness of the DETE concept for more complex PDEs, that are coupled and quasilinear.

In this study, the grid independent solution was generated by using 641 x 321 grid points. Since the oblique shock wave is a weak solution involving a sharp discontinuity, the solution generated on this or any other grid will clearly not be grid independent, but simply one selected to be sufficiently good. The solution obtained on this grid is used to compute the residuals $\left(R_{g}\right)$ in the DETEs (Eq. (23) and to calculate the actual absolute and relative errors, which can be used to assess how well DETEs predict errors on coarse meshes.

Figures 2 to 5 show the residual $\left(\mathrm{R}_{\mathrm{g}}\right)$ for the solutions generated on the following four coarser meshes: $321 \mathrm{x}$ $161,161 \times 81,81 \times 41,41 \times 21$. Only the residuals for the continuity equation (density) are shown. These residuals were computed by substituting the grid-independent solution into Eq. (22) with the coefficients A, B, and V computed by using the information on the coarse meshes. Thus, the residuals shown are exact. As expected, these figures show the residual to be high just upstream or downstream of the oblique shock. The blobs and jaggedness in the figures are due to the interpolation of the scheme used to plot contours.

Figure 6 shows the errors in the solutions generated on the coarsest mesh: $41 \times 21$. Only the relative errors in the computed solution for pressure and Mach number are shown. These errors were computed by using Eq. (23b); that is by subtracting the coarse mesh solution from the grid-independent solution at the center of each cell in the coarse mesh and then dividing by the grid-independent solution (e.g., $E_{r e}=\left|M_{g}-M_{c}\right| / M_{g}$ ). Trilinear interpolation is used to transfer the grid-independent solution to the center of each cell in the coarse mesh.

To demonstrate the usefulness of the DETEs derived, Eq. (23) with the exact $\mathrm{R}_{\mathrm{g}}$ (e.g., those shown in Figs. 2(a) to 5(a)) were used to compute the grid-induced error. These computations show that the DETEs were able to predict perfectly the errors if the $\mathrm{R}_{\mathrm{g}}$ is exact. Thus, this result is similar to what was found when testing the DETE concept on simpler model equations.

Since the residual $R_{g}$ is generally unknown, the main challenge in using DETE concept is to develop models for the residual. This effort is currently on-going. Also, the DETE concept was tested on meshes with triangular cells. These results can be obtained by e-mailing tomshih@iastate.edu.

\section{SUMMARY}

In this study, the discrete error-transport equation (DETE) is derived for the two-dimensional, compressible, Euler equations solved by a cell-centered finite-volume method. Results obtained show that DETE concept to be applicable to coupled, quasi-linear PDEs with weak solutions. More work is still needed in modeling the residual in the DETEs.

\section{REFERENCES}

1. Roache, P., Verification and Validation in Computational Science and Engineering, Hermosa Publishers, Albuquerque, New Mexico, 1998.

2. Oberkampf, W.L., Blottner, F.G., and Aeshliman, D., "Methodology for Computational Fluid Dynamics Code Verification and Validation," AIAA Paper 952226, June 1995.

3. Cosner, R.R., Oberkampf, W.L., Rahaim, C.P., Shih, T.I-P., "AIAA Committee on Standards for Computational Fluid Dynamics - Status and Plans," AIAA Paper 2004-00654, January 2004.

4. Carey, G.F., Computational Grids: Generation, Adaptation, and Solution Strategies, Taylor \& Francis, Washington, DC, 1997.

5. Celik, I., Chen, C.J., Roache, P.J., and Scheuer, G., Editors, Symposium on Quantification of Uncertainty in Computa-tional Fluid Dynamics, FED-Vol. 158, ASME Fluids Engineering Division, Summer Meeting, Washington D.C., 1993.

6. Celik, I. and Zhang, W.-M., "Calculation of Numerical Uncertainty Using Richardson Extrapolation: Application to Some Simple Turbulent Flow Calculations," ASME Journal of Fluids Engineering, Vol. 117, September 1995, pp. 439-445.

7. Wilson, R.V. and Stern, F., "Verification and Validation for RANS Simulation of a Naval Surface Combatant," AIAA Paper 2002-0904, Jan. 2002.

8. Peraire, J., Vahdati, M., Morgan, K., and Zienkiewicz, O.C., "Adaptive Remeshing for Compressible Flow Calculations," J. of Computational Physics, Vol. 22, 1976, pp.131-149.

9. Babuska, I. And Rheinboldt, W., "Error Estimates for Adaptive Finite Element Computations," SIAM Journal on Numerical Analysis, Vol. 15, No. 4, 1978, pp. 736-754.

10. Zienkiewicz, O.C. and Zhu, J.Z., "A Simple Error Estimator and Adaptive Procedure for Practical Engineering Analysis," International J. for Numerical Methods in Engineering, Vol. 24, 1987, pp. 337-357.

11. Sonar, T., "Strong and Weak Norm Refinement Indicators Based on the Finite Element Residual for Compressible Flow Computations," Impact of Computing in Science and Engineering, Vol. 5, 1993, pp. 111-127.

12. Oden, J.T., "Error Estimation and Control in Computational Fluid Dynamics," Mathematics of Finite Elements and Applications, edited by J.R. Whiteman, John Wiley and Sons, New York, 1994, Chapter 1.

13. Mackenzie, J., Sonar, T., and Suli, E., "Adaptive Finite Volume Methods for Hyperbolic Problems," Mathematics of Finite Elements and Applications, 
edited by J.R. Whiteman, John Wiley and Sons, New York, 1994, Chapter 19.

14. Venditti, D.A. and Darmofal, D.L., "Adjoint Error Estimation and Grid Adaptation for Functional Outputs: Application to Quasi-One-Dimensional Flow," Journal of Computational Physics, Vol. 164, 2000, pp. 204-227.

15. Shih, T.I-P., Gu, X., and Chu, D., "Grid-Quality Measures and Error Estimates," in Numerical Grid Generation in Computational Field Simulations, B.K. Soni, J.F. Thompson, J. Hauser, and P.R. Eiseman, editor, Mississippi State University, ISSG, 2000, pp. 799-808.

16. Gu, X., Schock, H.J., Shih, T.I-P., Hernandez, E.C., Chu, D., Keller, P.S., and Sun, R.L., "Grid-Quality Measures for Structured and Unstructured Meshes," AIAA Paper 2001-0652, Jan. 2001.

17. Gu, X. and Shih, T.I-P., "Differentiating between Error Source and Error Location in Solution-Adaptive Mesh Refinement," AIAA Paper 2001-2660, CFD Conference, June 2001.

18. Babuska, I., Strouboulis, T., and Upadhyay, C.S., "A Model Study of the Quality of a Posteriori Error Estimatort for Linear Elliptic Problems: Error Estimation in the Interior of Patchwise Uniform Grid of Triangles," Computer Methods in Applied Mechanics and Engineering, Vol. 114, 1994, pp. 307378.

19. Babuska, I., Strouboulis, T., Gangaraj, S.K., and Upadhyay, C.S., "Pollution Error in the h-Version of the Finite Element Method and Local Quality of the Recovered Derivatives," Computer Methods in Applied Mechanics and Engineering, Vol. 140, 1997, pp. 1-37.

20. Ferziger, J.H., "Estimation and Reduction of Numerical Error," in Symposium on Quantification of Uncertainty in Computa-tional Fluid Dynamics, FEDVol. 158, ASME Fluids Engineering Division, Summer Meeting, Washington D.C., 1993, pp. 1-8.

21. van Straalen, B.P., Simpson, R.B., and Stubley, G.D., "A Posteriori Error Estimation for Finite-Volume Simulations of Fluid Flow Transport," Proceedings of the $3^{\text {rd }}$ Annual Conference of the CFD Society of Canada, Vol. 1, Baniff, Alberta, June 1995.

22. Zhang, X.D., Trépanier, J.-Y., and Camarero, R., “A Posteriori Error Estimation for Finite-Volume Solutions of Hyperbolic Conservation Laws," Computational Methods in Applied Mechanics and Engineeering, Vol. 185, 2000, pp. 1-19.

23. Zhang, X.D., Pelletier, D., Trépanier, J.-Y., and Camarero, R., "Numerical Assessment of Error Estimators for Euler Equations," AIAA Journal, Vol. 39, No. 9, 2001, pp. 1706-1715.

24. Qin, Y. and Shih, T.I-P., "A Discrete Transport Equation for Error Estimation in CFD," AIAA Paper 2002-0906, January 2002.
25. Qin, Y. and Shih, T.I-P., "A Method for Estimating Grid-Induced Errors in Finite-Difference and FiniteVolume Methods," AIAA Paper 2003-0845, Jan. 2003.

26. Warming, R. F. and Hyett, B. J., "The Modified Equation Approach to the Stability and Accuracy Analysis of Finite-difference Methods," J. Comp. Physics \}, Vol. 14, 1974, pp. 159-179.

27. Celik, I., Hu, G., and Badeau, A., "Further Refinement and Bench Marking of a Single-Grid Error Estimation Technique," AIAA Paper 2003-0626, Jan. 2003.

28. Qin, Y. and Shih, T.I-P., "Analysis and Modeling of the Residual in the Discrete Error Transport Equation," AIAA Paper $2003-3850,16^{\text {th }}$ Computational Fluid Dynamics Conference, Orlando, June 2003.

29. Anderson, A., Modern Compressible Flow, with Historical Perspective, $2^{\text {nd }}$ Edition, McGraw-Hill Publishing Company, 1990.

30. Wang, Z.J., "Spectral (Finite) Volume Method for Conservation Laws on Unstructured Grids: Basic Formulation," J. Computational Physics, Vol. 178, pp. 210-251, 2002.

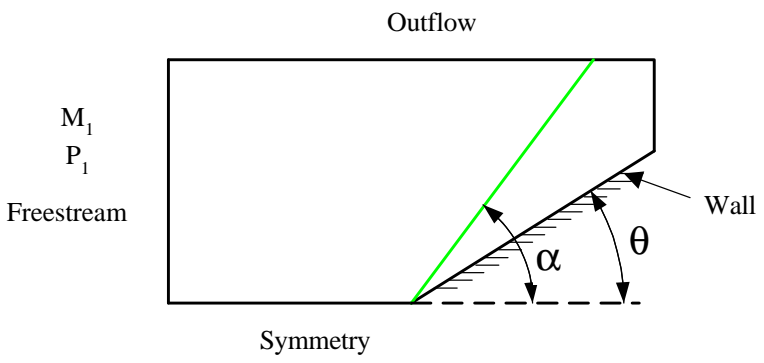

Fig. 1. Schematic of Problem. 


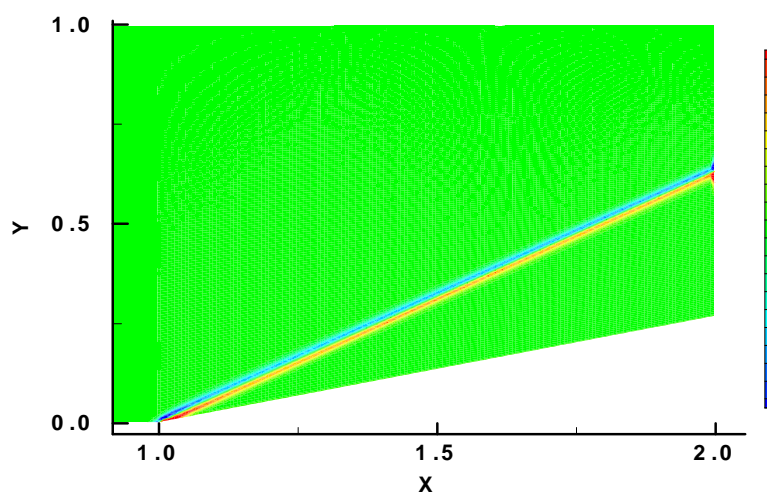

4.47E+03 $3.42 \mathrm{E}+03$ $2.37 \mathrm{E}+03$ $1.32 \mathrm{E}+03$ $2.63 \mathrm{E}+02$ $-7.89 E+02$ $-1.84 \mathrm{E}+03$ $-2.89 E+03$ $-3.95 E+03$ $-5.00 E+03$

Fig. 2. Exact Residual $\mathrm{R}_{\mathrm{g}}$ for the continuity equation: 321 x 161
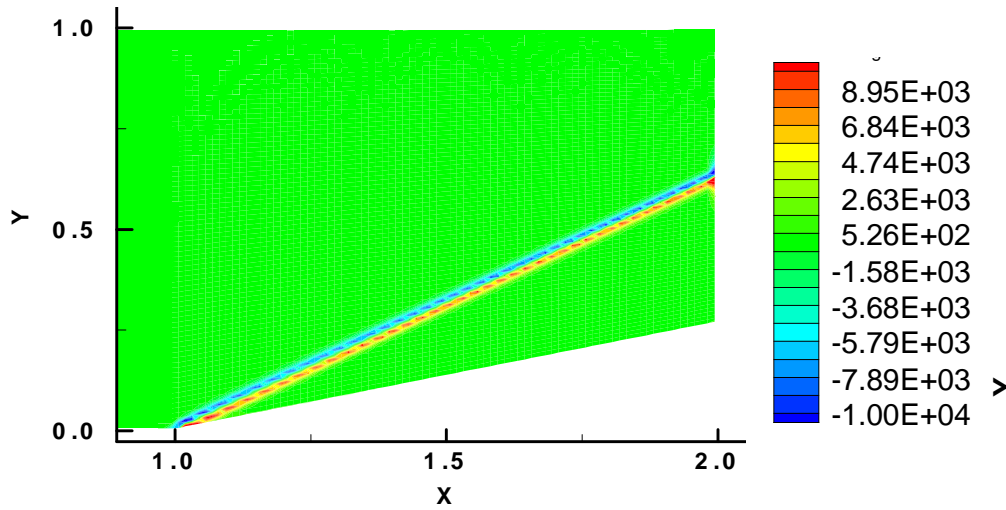

Fig. 3. Exact Residual $\mathrm{R}_{\mathrm{g}}$ for the continuity equation: $161 \mathrm{x} 81$

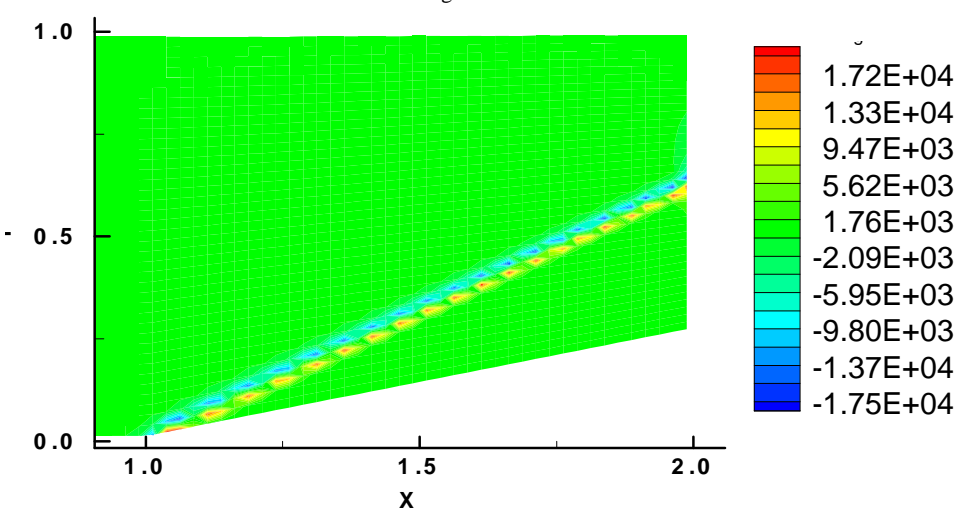

Fig. 4. Exact Residual $R_{g}$ for the continuity equation: $81 \times 41$

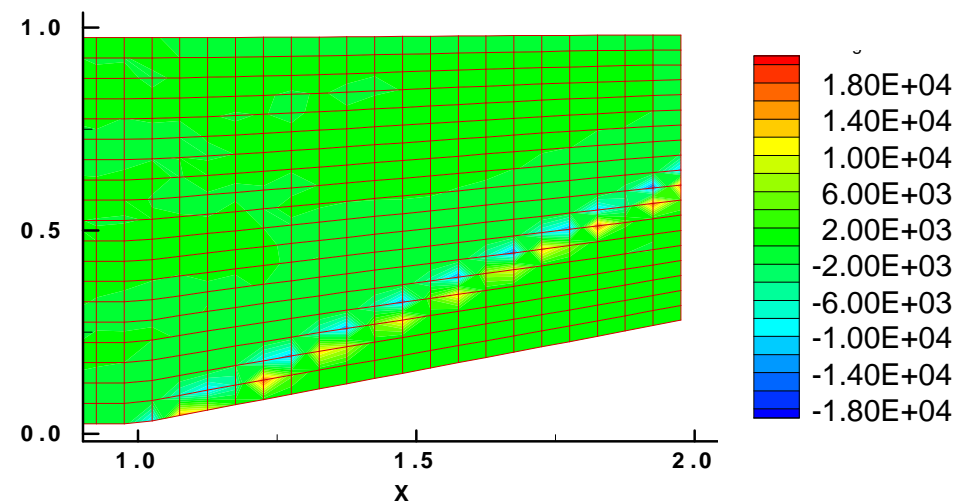

Fig. 5. Exact Residual $R_{g}$ for the continuity equation: 41 x 21
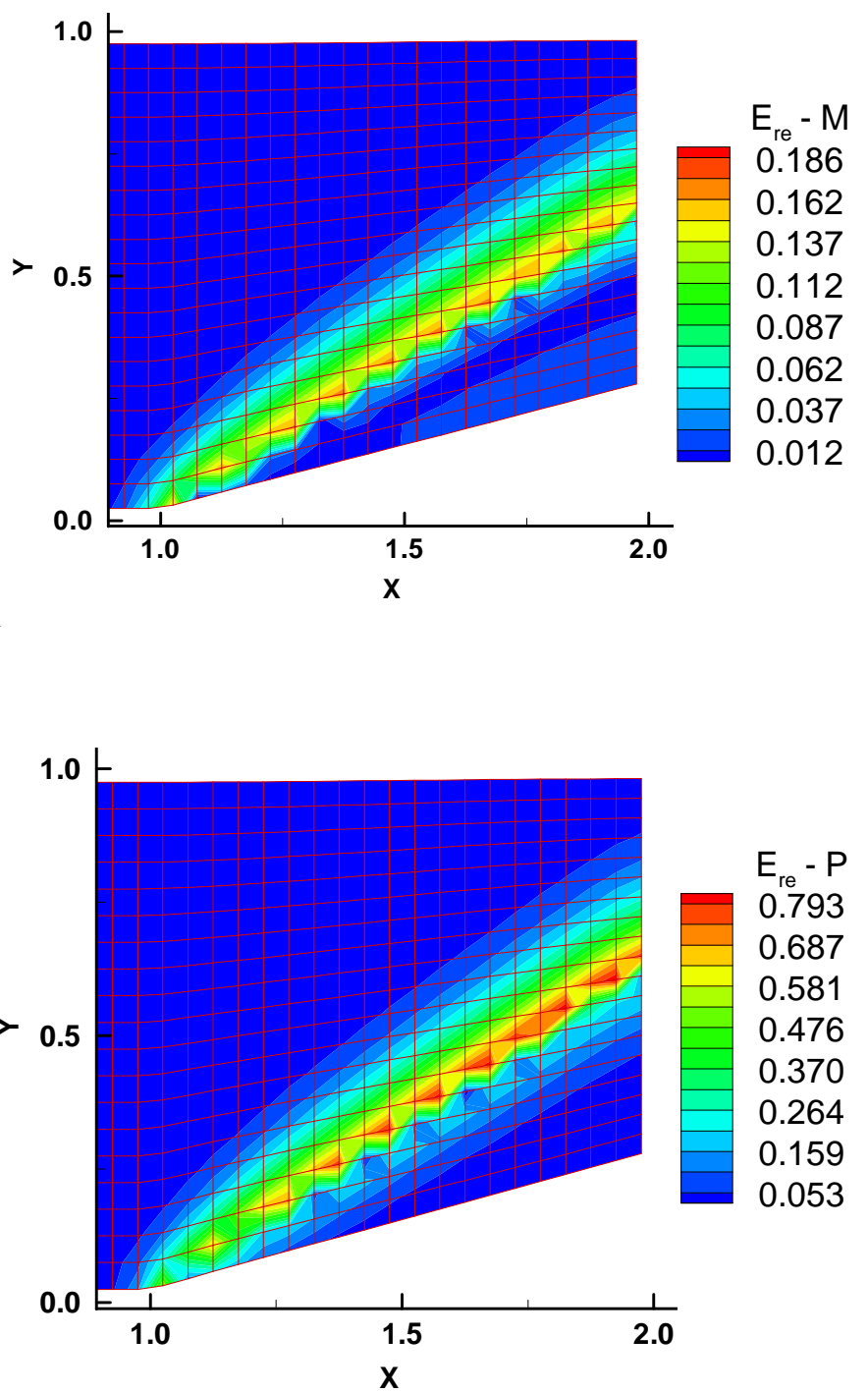

Fig. Actual relative error in solution generated on coarsest mesh: 41 x 21. Top: Mach number. Bottom: pressure. 